\title{
Dispersion corrections in graphenic systems: a simple and effective model of binding
}

\author{
Tim Gould, ${ }^{1}$ S. Lebègue, ${ }^{2}$ and John F. Dobson ${ }^{1,2}$ \\ ${ }^{1}$ Queensland Micro and Nano Technology Centre, \\ Griffith University, Nathan, Queensland 4111, Australia \\ ${ }^{2}$ Laboratoire de Cristallographie, Résonance Magnétique et Modélisations (CRM2, \\ UMR CNRS 7036) Institut Jean Barriol, Université de Lorraine BP 239, \\ Boulevard des Aiguillettes 54506 Vandoeuvre-lès-Nancy, France
}

\begin{abstract}
We combine high-level theoretical and ab initio understanding of graphite to develop a simple, parametrised force-field model of interlayer binding in graphite, including the difficult nonpairwise-additive coupled-fluctuation dispersion interactions. The model is given as a simple additive correction to standard density functional theory (DFT) calculations, of form $\Delta U(D)=$ $f(D)\left[U^{\mathrm{vdW}}(D)-U^{\mathrm{DFT}}(D)\right]$ where $D$ is the interlayer distance. The functions are parametrised by matching contact properties, and long-range dispersion to known values, and the model is found to accurately match high-level $a b$ initio results for graphite across a wide range of $D$ values. We employ the correction on the difficult bigraphene binding and graphite exfoliation problems, as well as lithium intercalated graphite $\mathrm{LiC}_{6}$. We predict the binding energy of bigraphene to be $0.27 \mathrm{Jm}^{-2}$, and the exfoliation energy of graphite to be $0.31 \mathrm{Jm}^{-2}$, respectively slightly less and slightly more than the bulk layer binding energy $0.295 \mathrm{Jm}^{-2} /$ layer. Material properties of $\mathrm{LiC}_{6}$ are found to be essentially unchanged compared to the local density approximation. This is appropriate in view of the relative unimportance of dispersion interactions for $\mathrm{LiC}_{6}$ layer binding.
\end{abstract}

PACS numbers: 71.15.Nc,71.45.Gm,81.05.uf 


\section{INTRODUCTION}

Interlayer binding of graphenic structures such as bigraphene and graphite is very challenging to evaluate in ab initio calculations. Standard approaches like the local density ${ }^{1}$ and generalized gradient ${ }^{2-4}$ approximations (LDA and GGA), and van der Waals (vdW) density functional theories (vdW DFTs eg. vdW-DF, ${ }^{5-7}$ TS-approach, ${ }^{8}$ DFT-D ${ }^{* 9-11}$ and VV10; ${ }^{12}$ see Ref. 13 for an overview) either miss (LDA/GGA) or mispredict ${ }^{14}$ (vdW DFT) the dispersive binding, especially in the asymptotic limit. This failure is in part due to the presence $^{15}$ of coupled long-wavelength charge fluctuations, which influence the dispersion interactions between graphene layers, ${ }^{16}$ and which are difficult to predict via conventional means.

The random-phase approximation to the adiabatic connection, fluctuation-dissipation functional approach ${ }^{17,18}$ (ACFD-RPA or RPA) seamlessly predicts the dispersive binding of graphite. ${ }^{19}$ The direct RPA includes all plasmon interactions, and is known ${ }^{20}$ to accurately predict the binding properties of a wide variety of systems. However RPA is computationally difficult to evaluate for graphenic materials, requiring substantial computer resources even for the relatively simple case of bulk graphite. ${ }^{19}$ For example the RPA binding energy of bigraphene and exfoliation energy of graphite (the energy required to remove a single layer from the surface of bulk graphite) are difficult to evaluate with present computational resources, although sophisticated attempts ${ }^{14}$ have been made to predict both through rescaled dispersion corrections.

A simple way to deal with dispersion is to use efficient dispersion-free calculations (LDA/GGA) and simply add a model potential accounting for the difficult vdW properties. Such an approach is similar to the popular DFT-D*9-11 and Tkatchenko-Scheffler $(\mathrm{TS})^{8}$ vdW approximations. Previously there have been many attempts ${ }^{21-25}$ to develop semiempirical model potentials using the known properties of bulk graphite. Until recently, however, reasonable values of certain material properties like the interlayer binding energy $\left[\epsilon_{b} \equiv E(D)-E(\infty)\right.$ normalised per atom or by area where $E(D)$ is the energy of bulk graphite with intra-layer atomic coordinates fixed and all graphene layers a distance $D$ from their nearest neighbours] and the inelastic interlayer coefficient $\left[C_{333} \propto d_{3} / d D^{3} E\left(D_{0}\right)\right]$ were not available. Additionally, many early attempts did not include the unusual van der Waals

properties $^{16,25-27}$ of graphenic structures, and thus incorrectly reproduced the intermediate- 
and long-distance binding.

In this work we will use the latest theoretical results, and $a b$ initio data from accurate ACFD-RPA calculations to develop a model potential for graphite. The model will be simple to evaluate and to use. To ensure physical realism we shall ensure that the model satisfies many constraints, namely the first three energy derivatives at the optimal lattice spacing, and the vdW dispersion potential away from contact. The model can be used on its own in force field modelling, or to supplement LDA and GGA calculations, with the LDA recommended over the GGA due to its better predictive power.

The model potential will then be extended to the cases of bigraphene and graphite exfoliation, and used to predict the important binding and exfoliation energies, and other properties. Finally the model potential will be tested on lithium intercalated graphite $\left(\mathrm{LiC}_{6}\right)$. We shall show that the model gives reasonable results for all cases, and should thus be employable on a wide range of graphenic systems.

\section{A NOTE ON ENERGY UNITS}

In this paper we compare a number of different graphitic systems. Special care must be taken when considering energy properties, as these can become ambiguous when comparing between different geometrical arrangements. These issues are discussed as they arise in the text, but we raise some here to help avoid confusion.

Where direct comparisons are made between different geometries (such as in Table II) we use $\mathrm{Jm}^{-2}$ which is the energy divided by the surface area of a single layer for bigraphene and graphite exfoliation, and the energy divided by the surface area divided by the number of layers for the bulk systems graphite and lithium intercalated graphite. The elastic coefficient $C_{33}$ is defined in the standard way for graphite and $\mathrm{LiC}_{6}$. For bigraphene and exfoliation there is no natural way to define $C_{33}$ and we define it via the second derivative, with respect to the outermost layer spacing, of the energy per area, scaled appropriately to allow comparison with bulk graphite.

We also give results in meV/Atom as this is a natural unit for bulk systems, especially for $a b$ initio studies. Here we take the total energy of a cell divided by the number of carbon atoms in the cell. The one exception is in the exfoliation calculations, where we consider only the atoms in the two layers forming the opened surface (four atoms for the 
standard $\mathrm{AB}$ cell used here). For carbon atoms with in-plane C-C bond length $a_{0}$ given in Angstrom we convert energy $\epsilon$ from meV/Atom to $\mathrm{Jm}^{-2}$ via $\epsilon\left[\mathrm{Jm}^{-2}\right]=S \epsilon[\mathrm{meV} /$ Atom $]$ where $S_{\text {graphite }}=S_{\mathrm{LiC}_{6}}=0.006158 \times 2 / a_{0}^{2}$ for the bulks and $S_{\text {bigraphene }}=S_{\text {exfoliation }}=0.006158 \times 4 / a_{0}^{2}$ for the layer pairs.

\section{NEAR-CONTACT PROPERTIES OF GRAPHITE}

Key to previous models of graphene binding are three material properties: the lattice constant $c$ of $\mathrm{AB}$ graphite (or interlayer distance $D_{0}$ where $c=2 D_{0}$ ), the inter-planar elastic coefficient $C_{33}$, and the binding energy $\epsilon_{b}$. These are listed in order of experimental inconsistency, with the binding energy showing the greatest variation across different experiments.

The lattice constant $c$ has been found ${ }^{28}$ very accurately to be $c=6.68 \AA$ corresponding to an inter-layer distance $D_{0}=3.34 \AA$, while the elastic coefficient has been narrowed down by experiment ${ }^{29-32}$ to a small range $C_{33} \approx 38.6 \pm 2.1 \mathrm{GPa}$. The binding energy has never been directly measured, and most efforts ${ }^{33-36}$ to determine it involve unreliable theoretical models ${ }^{37}$ of dispersion to reverse engineer the binding energy from other properties. Estimates range between about $25 \mathrm{meV} /$ Atom $=0.15 \mathrm{Jm}^{-2} /$ layer to $57 \mathrm{meV} /$ Atom $=0.35 \mathrm{Jm}^{-2} /$ layer. Until recently, theory has fared little better than experiment at prediciting $\epsilon_{b}$. Here eg. the GGA predicts a binding energy of $2.3 \mathrm{meV} /$ Atom, ${ }^{21}$ the LDA predicts $24 \mathrm{meV} /$ Atom, ${ }^{19}$ and different vdW corrected DFT approaches ${ }^{5-12}$ predict $^{14,38,39}$ values between 24 (layer vdW-DF ${ }^{6}$ ) and $71 \mathrm{meV} /$ Atom $\left(\mathrm{VV} 10^{14}\right)$.

The authors et al. ${ }^{19}$ previously published accurate RPA calculations for graphite. The RPA is known ${ }^{20}$ to be very good at predicting many properties of bulk systems, and in the absence of conclusive experiments we consider it to be a benchmark for graphite energy differences. In those calculations, the binding energy was found to be $\epsilon_{b}=48 \mathrm{meV} / \mathrm{Atom}=$ $0.295 \mathrm{Jm}^{-2} /$ layer, comparable to $\epsilon_{b}=56 \pm 5 \mathrm{meV} /$ Atom found through high-level quantum Monte-Carlo (QMC) calculations. ${ }^{40}$ Unlike QMC, RPA also gave a lattice distance $D_{0}=$ $3.34 \AA$ and an elastic constant $C_{33}=36 \mathrm{GPa}$, in excellent agreement with experiment.

For this work, additional RPA calculations were performed using the same parameters as Ref. 19 at additional inter-layer distances $D$. Using the extra points we can now calculate 
the inelastic constant $C_{333}=-530 \mathrm{GPa} \pm 10 \%$, where $C_{333}$ is defined via

$$
\frac{F_{3}(D)}{V_{0}} \underset{D \approx D_{0}}{\approx} C_{33}\left(\frac{D}{D_{0}}-1\right)+C_{333}\left(\frac{D}{D_{0}}-1\right)^{2}
$$

(where $F_{3} / V_{0} \equiv \frac{D}{D_{0}} \frac{\mathrm{d} E}{\mathrm{~d} D}$ is the normalised force per unit area required to distort graphite in the inter-planar direction $\vec{a}_{3}$ ) so that

$$
C_{33}=\left.\frac{D_{0}^{2}}{V_{0}} \frac{d^{2} E(D)}{d D^{2}}\right|_{D_{0}}, \quad C_{333}=\left.\frac{D_{0}^{3}}{2 V_{0}} \frac{d^{3} E(D)}{d D^{3}}\right|_{D_{0}} .
$$

Here $D_{0}$ is the equilibrium lattice distance, $V_{0}$ is the equilibrium volume of the unit cell and $E$ is the energy of the graphene unit cell with lattice parameters $a=a_{0}$ and $c=2 D_{0}$. For reference we note that $\mathrm{AB}$ graphite has an optimal unit cell defined by $\vec{a}_{1}=a_{0}(\sqrt{3} / 2,3 / 2,0)$, $\vec{a}_{2}=a_{0}(-\sqrt{3} / 2,3 / 2,0)$ and $\vec{a}_{3}=2 D_{0}(0,0,1)$ where $a_{0}$ is the C-C distance in the plane $\approx 1.42 \AA$ so that the unit cell has volume $V_{0}=\sqrt{27} a_{0}^{2} D_{0}$.

From the RPA data we thus have four, well-converged constraints on any model function of graphite: the inter-layer distance $D_{0}$, the binding energy $\epsilon_{b}$, and the elastic and inelastic constants $C_{33}$ and $C_{333}$. A model which reproduces all four should be expected to reproduce the near-contact behaviour of graphite, at least at the RPA level.

\section{VDW DISPERSION POTENTIAL OF GRAPHITE}

In addition to the near-contact behaviour, we also seek a model which can reproduce the asymptotic dispersion properties. Dispersion in graphite is comprised of two major contributions: i) 'unusual' coupled-fluctuation graphenic interactions from the gapless transitions between $\pi_{z}$ and $\pi_{z}^{*}$ orbitals; and ii) 'usual' insulating van der Waals interactions arising from all other transitions. The former have been previously ${ }^{16,25}$ shown to give rise to a potential with the asymptotic form $-C^{3} / D^{3}$. The authors more recently showed ${ }^{27}$ that this

form is only valid at quite large distances, with $-C_{3} \frac{2}{\pi} \operatorname{atan}\left(D / D_{c}+\phi_{c}\right) / D^{3}$ being more valid at intermediate values. The latter give rise to a potential with the conventional 'planar' formula $-C_{4} / D^{4}$, whose form is consistent with sums over the layers of inter-atomic interactions $-C_{6} / R^{6}$. There is evidence ${ }^{41}$ that even here the conventional summing technique is problematic. We avoid any non-additivity issues by fitting to the geometry dependent RPA results and bypassing less reliable sums over atoms. 
FIG. 1. Model vdW and ACFD-RPA correlation energies of graphite as a function of interlayer distance.

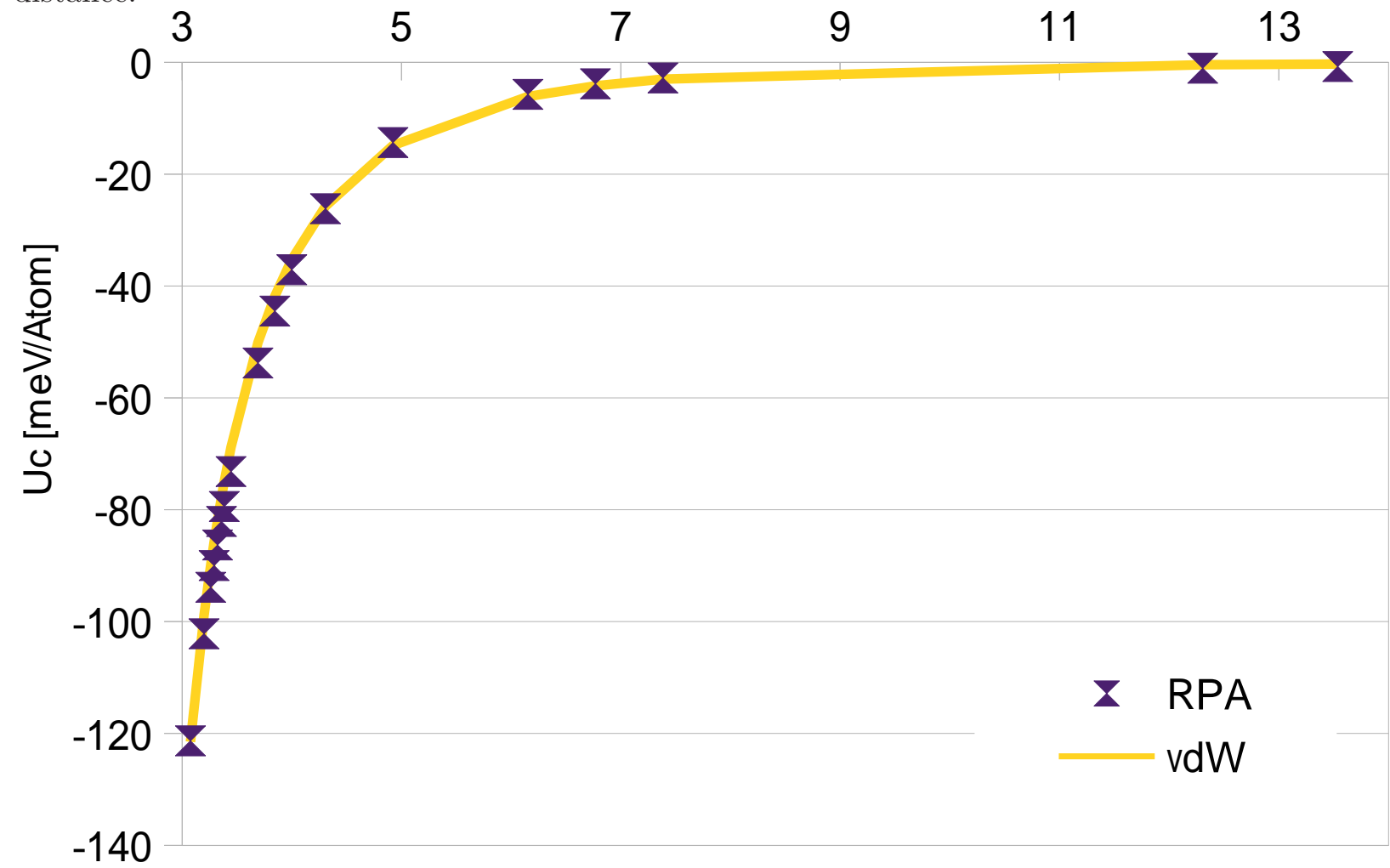

\section{$\mathrm{D}[\mathrm{Ang}]$}

To leading orders in $1 / D$, the dispersion energy per carbon atom can thus be written as

$$
\begin{aligned}
U^{\mathrm{vdW}}(D) & \equiv \frac{E^{\mathrm{vdW}}(D)}{N_{\mathrm{C}}} \underset{D \gg D_{0}}{\approx} U^{(3)}(D)+U^{(4)}(D) \\
U^{(3)}(D) & =\frac{-C_{3}}{D^{3}} \frac{2}{\pi} \operatorname{atan}\left(\frac{D}{D_{c}}+\phi_{c}\right) \\
U^{(4)}(D) & =\frac{-C_{4}}{D^{4}-D_{s}^{4}}
\end{aligned}
$$

where $D_{s}$ accounts for higher order corrections. Here $C_{3}, D_{c}$ and $\phi_{c}$ are determined by the properties of graphite's Dirac cones, but we must determine $C_{4}$ and $D_{s}$ via best fits to $a b$ initio ACFD-RPA energies.

The parameters $D_{c}$ and $\phi_{c}$ depend on an assumed cutoff energy for interactions between the $\pi_{z}$ and $\pi_{z}^{*}$ Dirac cones, as well as the Fermi velocity. Using the LDA Fermi velocity of $850 \mathrm{kms}^{-1}$, and assuming a physically plausible energy cutoff of $5 \mathrm{eV}^{42}$ gives $^{27} C_{3}=0.38$, $D_{c}=23.7 \AA$ and $\phi_{c}=0.62$, and we use these parameters henceforth. This allows a fitting to be made on the ACFD-RPA correlation energy $U_{\mathrm{c}}^{\mathrm{RPA}}$ to determine $C_{4}$ and $D_{s}$. Here we 
seek to minimise

$$
\operatorname{Err}(D)=\left[U_{\mathrm{c}}^{\mathrm{RPA}}(D)-U^{(3)}(D)+\frac{C_{4}}{D^{4}-D_{s}^{4}}\right]
$$

with respect to $C_{4}$ and $D_{s}$ according to some metric. As shown in Figure 1 we find $D_{s}=$

$2.22 \AA$ and $C_{4}=7.57 \mathrm{eV}^{-4}$ give a good fit to the RPA correlation energy across $3<D<$ $13 \AA$. For convenience, all best-fit parameters including these are presented in Table I.

\section{MODEL CORRECTION}

Hasegawa and Nishidate ${ }^{21}(\mathrm{HN})$ suggested that a model of graphite interactions could be developed around the assumption that standard groundstate DFT (LDA/GGA) is valid in the inner $D<D_{0}$ region, while the dispersion potential dominates for $D>D_{0}$, and thus a fit between these two functions would be appropriate for the total energy. It is in this spirit that we develop our model. With the aim of simplicity we follow the ideas of their earlier work $^{21}$ more than those of their latter. ${ }^{22}$

$\mathrm{HN}$ found that the potential energy of the local density approximation (LDA) or generalised gradient approximation (GGA) was well-reproduced by a function

$$
\begin{aligned}
U^{\mathrm{DFT}}(D) & \approx M(D / \tilde{D}-1) \\
M(x) & = \begin{cases}-M_{0} \frac{\tau_{2} e^{-\tau_{1} x}-\tau_{1} e^{-\tau_{2} x}}{\tau_{2}-\tau_{1}}, & \tau_{1} \neq \tau_{2} \\
-M_{0}(1+\tau x) e^{-\tau x}, & \tau_{1}=\tau_{2}=\tau\end{cases}
\end{aligned}
$$

(the second case is the limit $\tau_{1} \rightarrow \tau_{2}$ of the first) where $U^{\mathrm{DFT}}(D)$ takes its minimum at $D=\tilde{D}$. HN found $M_{0}=26.5 \mathrm{meV}, \tilde{D}=3.311 \AA$ and $\tau=8.065$ accurately reproduced their LDA calculations for graphite. We evaluated the graphite binding curve using VASP ${ }^{43,44}$ calculations with a cutoff energy of $700 \mathrm{eV}$, in-plane C-C distance $1.421 \AA$ and a $\vec{k}$-grid of $24 \times 24 \times 4$ points including the $\Gamma$ point, and found that the similar $M_{0}=25.4 \mathrm{meV}$, $\tilde{D}=3.318 \AA$ and $\tau=8.157$ gave slightly better agreement (under $0.3 \mathrm{meV}$ maximum absolute error). We use the new parameters in this work. The HN parameters for PW912 GGA results were $M_{0}=2.3 \mathrm{meV}, \tilde{D}=4.407, \tau_{1}=2.523$ and $\tau_{2}=12.99$, and these provide a decent approximation to $\mathrm{PBE}^{4}$ data from VASP. However given its poor binding parameters, and high sensitivity to the in-plane lattice constant, ${ }^{45}$ we do not recommend using GGA in graphenic systems, and include it mostly for illustrative purposes. 
Following HN we model the dispersion-corrected energy via

$$
\begin{aligned}
U(D) & \approx U^{\mathrm{Calc}}(D)+\Delta U(D) \\
\Delta U(D) & =f\left(\frac{D}{D_{0}}-1\right)\left[U^{\mathrm{vdW}}(D)-U^{\mathrm{DFT}}(D)\right]
\end{aligned}
$$

where $U^{\text {Calc }}(D)$ is the potential energy calculated via lower-level LDA/GGA theory, $U^{\mathrm{vdW}}(D)$ is defined in $(3)$ and $U^{\mathrm{DFT}}(D)$ is defined in $(7)$ and involves the same approximation (LDA or GGA) as $U^{\text {Calc }}$. To match all constraints we employ a four parameter fitting function

$$
f(x)=\left[1+\kappa e^{-\left(a_{1} x+a_{2} x^{2}+a_{3} x^{3}\right)}\right]^{-1} .
$$

Here $\kappa>0$ and $a_{3}>0$ are required to ensure $f(x \gg 0)=1$ so that the potential is dominated by dispersion for $D \gg D_{0}$.

We determine the best fit parameters $\mathcal{C}=\left\{\kappa, a_{1}, a_{2}, a_{3}\right\}$ by ensuring that the model correctly reproduces energy derivatives at the contact distance $D=D_{0}$ when the model DFT potential $U^{\mathrm{DFT}}$ is used to represent $U^{\text {Calc }}$ ie. we ensure that derivatives of $(1-f) U^{\mathrm{DFT}}+$ $f U^{\mathrm{vdW}}$ equal derivative of $U^{\mathrm{RPA}}$. The parameters are thus found via

$$
\left.\frac{d_{p}}{d D^{p}}[f(D ; \mathcal{C}) P(D)]\right|_{D_{0}}=\left.\frac{d_{p}}{d D^{p}} Q(D)\right|_{D_{0}}
$$

for $p=0 \ldots 3$ where $P(D)=U^{\mathrm{vdW}}(D)-U^{\mathrm{DFT}}(D)$ and $Q(D)=U^{\mathrm{RPA}}(D)-U^{\mathrm{DFT}}(D)$. Using the dispersion potential (3) and LDA/GGA model potential (7) with the parameters found earlier and tabulated in Table I we find $\kappa=1.420, a_{1}=12.5, a_{2}=-8.1$ and $a_{3}=137.5$ for the LDA and $\kappa=0.578, a_{1}=10.0, a_{2}=-7.8$ and $a_{3}=30.7$ for the GGA.

We test the model by adding appropriately parametrised $\Delta U(D)$ to $U^{\mathrm{LDA}}(D)$ and $U^{\mathrm{GGA}}(D)$ and comparing with the RPA. As can be seen in Figure 2 the appropriate models agree well with the RPA values across all $D$, using ab initio data from either the LDA or GGA. We note that the fitting involves matching three derivatives at the origin, and the asymptotic tail, so agreement at the intermediate points was not guaranteed. The agreement with the RPA is surprising for the GGA calculations, as without correction the GGA predicts a grossly inaccurate inter-layer distance $D_{0}^{\mathrm{GGA}}=4.407 \AA$ and binding energy $\epsilon_{b}^{\mathrm{GGA}}=2.3 \mathrm{meV} /$ Atom 
TABLE I. Best-fit parameters of all functions presented in this work. Parameters are for graphite, while the final row gives the geometry modifier for bigraphene. Most quantities are unitless except: $C_{3}$ is in $\mathrm{eV} \AA^{-3}, C_{4}$ is in $\mathrm{eV} \AA^{-4}, M_{0}$ is in $\mathrm{meV}, D_{c}, D_{s}$ and $\tilde{D}$ are in $\AA$.

\begin{tabular}{lllll}
\hline \hline$U^{\mathrm{vdW}}$ & $C_{3}=0.38$ & $D_{c}=23.7$ & $\phi_{c}=0.62$ & \\
& $C_{4}=7.57$ & $D_{s}=2.22$ & & \\
\hline$U^{\mathrm{LDA}}$ & $M_{0}=25.4$ & $\tilde{D}=3.318$ & $\tau=8.157$ & \\
$f^{\mathrm{LDA}}$ & $\kappa=1.420$ & $a_{1}=12.5$ & $a_{2}=-8.1$ & $a_{3}=137.5$ \\
\hline$U^{\mathrm{GGA}}$ & $M_{0}=2.3$ & $\tilde{D}=4.407$ & $\tau_{1}=2.523$ & $\tau_{2}=12.99$ \\
$f^{\mathrm{GGA}}$ & $\kappa=0.578$ & $a_{1}=10.0$ & $a_{2}=-7.8$ & $a_{3}=30.7$ \\
\hline $\mathrm{Bi}$ & $G_{3}=0.455$ & $G_{4}=0.462$ & $G_{L}=\frac{1}{2}$ & \\
\hline \hline
\end{tabular}

FIG. 2. Energy of graphite as a function of $D$ in the RPA, LDA/GGA and LDA/GGA with vdW dispersion corrections. GGA+vdW (maroon, fine dotted line) match LDA+vdW almost perfectly (red, solid line).

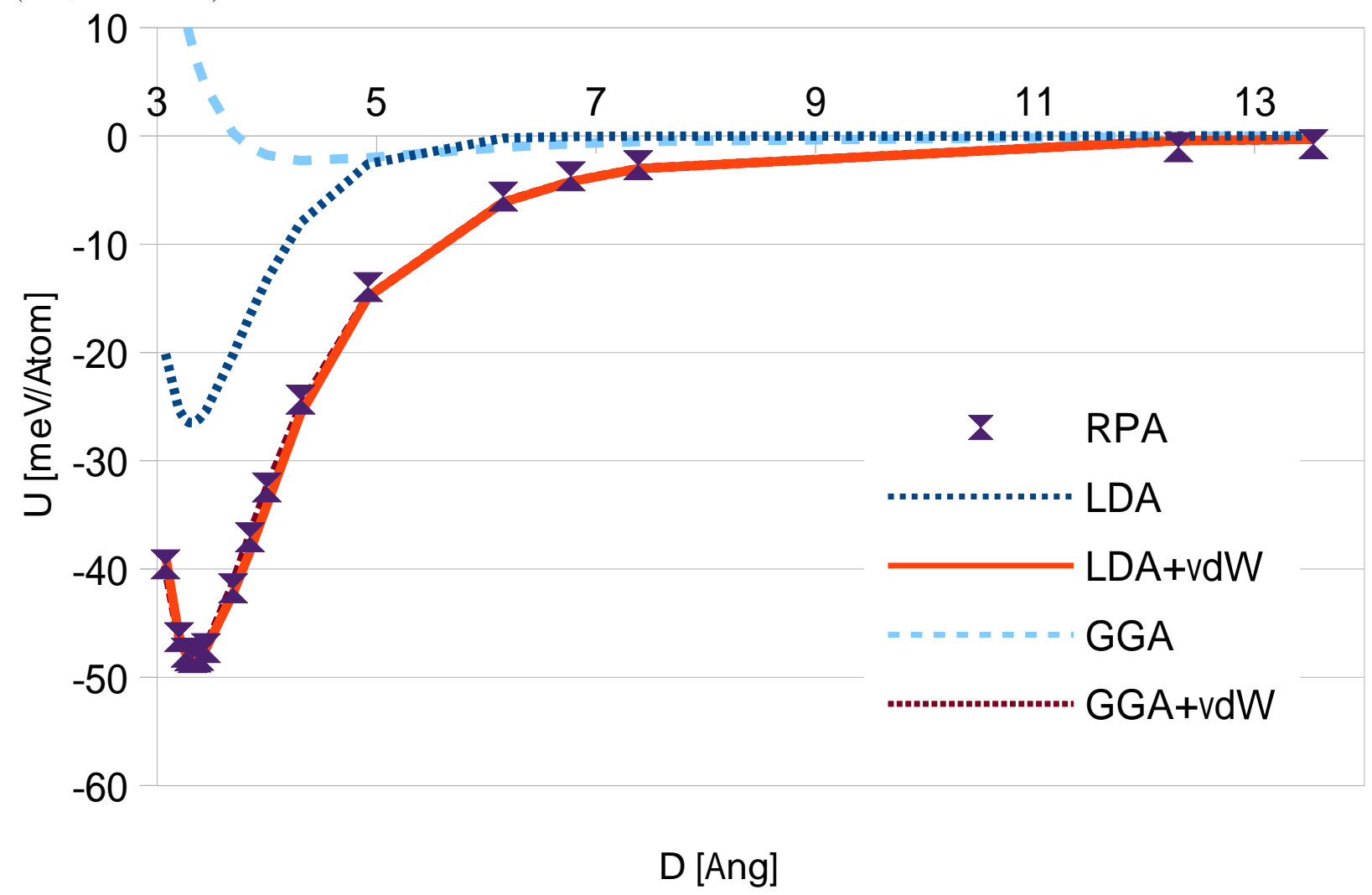




\section{BIGRAPHENE AND EXFOLIATION}

At the moment, RPA results are unavailable for bigraphene and exfoliation. We thus propose to use the modelled properties of graphite to predict properties of the more difficult systems. This requires some adjustment of the theory to take into account the different geometries, but otherwise follows the same broad approach.

It can be shown that for bigraphene the vdW dispersion and LDA fit takes the same basic form

$$
\begin{aligned}
U_{\mathrm{bi}}^{(3)}(D) & =\frac{-C_{3}^{\mathrm{bi}}}{D^{3}} \frac{2}{\pi} \operatorname{atan}\left(\frac{D}{D_{c}^{\mathrm{bi}}}+\phi_{c}^{\mathrm{bi}}\right) \\
U_{\mathrm{bi}}^{(4)}(D) & =\frac{-C_{4}^{\mathrm{bi}}}{D^{4}-D_{s}^{\mathrm{bi}^{4}}} \\
U_{\mathrm{bi}}^{\mathrm{DFT}}(D) & =M\left(\frac{D}{\tilde{D}^{\mathrm{bi}}}-1 ; \mathrm{bi}\right)
\end{aligned}
$$

[where $M\left(x\right.$; bi) is Eq. (7b) with bigraphene parameters $M_{0}^{\text {bi }}$ and $\tau_{1 / 2}^{\text {bi }}$ ] as for graphite, but with different parameters $C_{3}^{\mathrm{bi}}, D_{c}^{\mathrm{bi}}, \phi_{c}^{\mathrm{bi}}, C_{4}^{\mathrm{bi}}, D_{s}^{\mathrm{bi}}, \tilde{D}^{\mathrm{bi}}, M_{0}^{\mathrm{bi}}$ and $\tau_{1 / 2}^{\mathrm{bi}}$. In Appendix A we argue that the most important effect of changing the geometry from graphite to bigraphene are the changes to $C_{3}^{\mathrm{bi}}, C_{4}^{\mathrm{bi}}$ and $M_{0}^{\mathrm{bi}}$. Thus we can simply rescale the graphite functions so that equation (9) becomes

$$
\begin{aligned}
U_{\mathrm{bi}}(D)= & U_{\mathrm{bi}}^{\mathrm{Calc}}(D)+\Delta U_{\mathrm{bi}}(D) \\
\Delta U_{\mathrm{bi}}(D) \approx & f\left(\frac{D}{D_{0}}-1\right)\left[G_{3} U^{(3)}(D)+G_{4} U^{(4)}(D)\right. \\
& \left.-G_{L} U^{\mathrm{DFT}}(D)\right]
\end{aligned}
$$

for bigraphene. Here $G_{3}=0.455, G_{4}=1 /[2 \zeta(4)]=0.462$ and $G_{L}=\frac{1}{2}$ are the geometry factors for converting the graphite results to bigraphene. We assume that the fitting function is unchanged from its graphite form and parametrisation.

Using VASP ${ }^{43,44}$ we evaluate $U_{\mathrm{bi}}^{\mathrm{LDA}}(D)$ and add $(16)$ to introduce the dispersions. Here we use similar convergence parameters to bulk graphite, with an energy cutoff of $700 \mathrm{eV}$, C-C distance $1.421 \AA, \vec{k}$-grid of $24 \times 24 \times 1$. We perform all calculations in a supercell with $c=39.38 \AA$. The vaccuum between bigraphene units is always over $20 \AA$ so that contamination with neighbouring cells is avoided.

The LDA and dispersion-corrected LDA results are plotted in Figure 3, showing the importance of corrections to bigraphene. We find the binding distance to be unchanged 
FIG. 3. Energy of bigraphene and single-layer exfoliation as a function of $D$ in the LDA and LDA with vdW dispersion corrections. Includes correction alone.

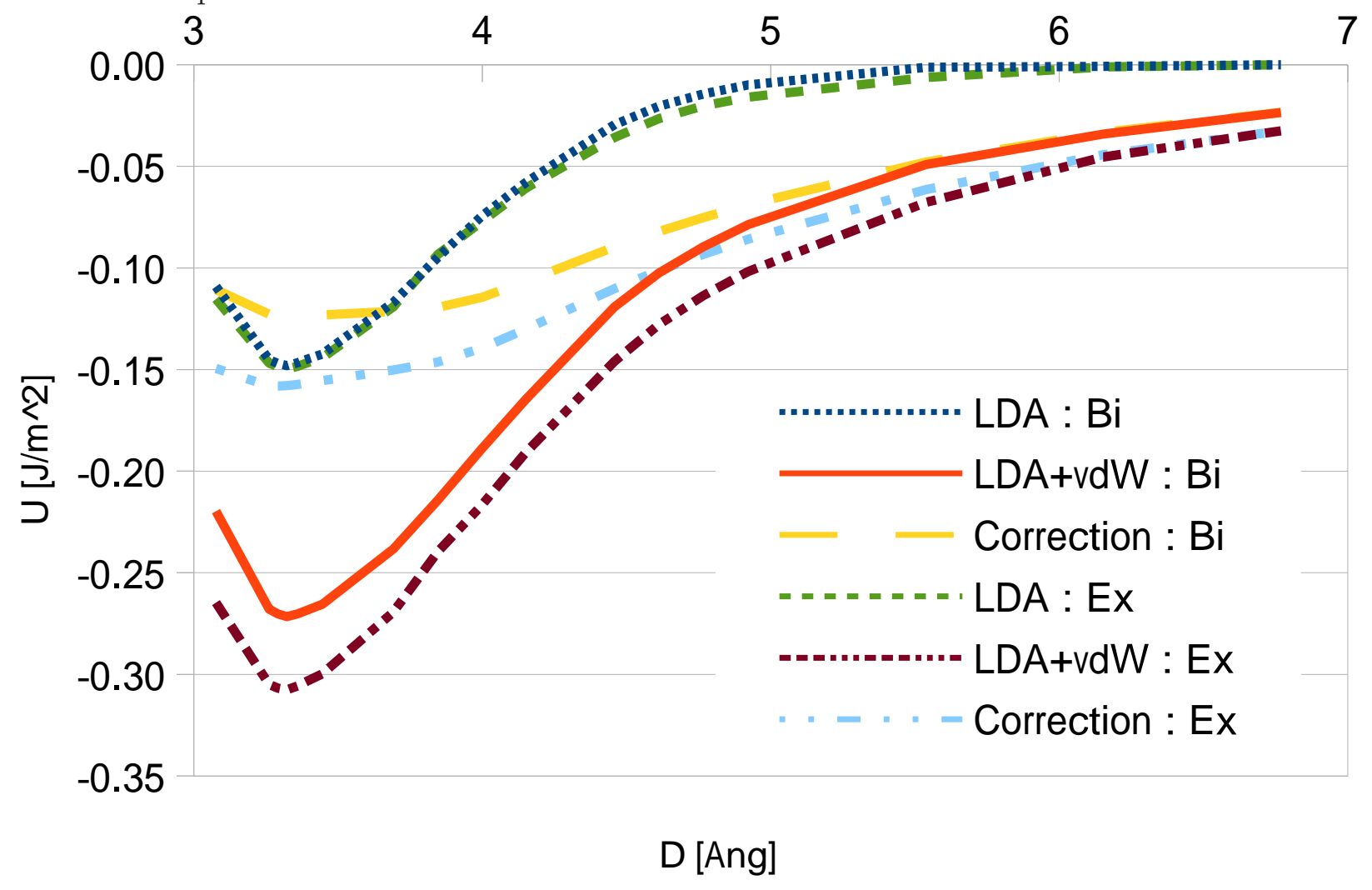

(to numerical accuracy) by the dispersion correction at $D_{0}^{\mathrm{LDA}} \approx D_{0}=3.32$, but we find a substantial change to the predicted binding energy, from $12 \mathrm{meV} /$ Atom in the LDA to $22 \mathrm{meV} /$ Atom with the correction. While $C_{33}$ is somewhat ill-defined for bigraphene, we find the effective coefficient $C_{33}=2 \frac{D_{0}^{2}}{V_{0}} \frac{d^{2} E(D)}{\mathrm{d} D^{2}}$ (the factor 2 allows direct comparison with graphite - each layer of bigraphene has only one nearest neighbour whereas layers of graphite have two) is increased by $22 \%$ compared to the LDA, with $C_{33}^{\mathrm{LDA}}=29 \mathrm{GPa}$ and $C_{33}=35 \mathrm{GPa}$. Converting the binding energy to be per unit of surface area $\left(\times 2 / A_{\mathrm{C}}\right.$ where $A_{\mathrm{C}}$ is the area per carbon atom), we see $\epsilon_{b}^{\mathrm{bi}}=22 \mathrm{meV} /$ Atom $=0.27 \mathrm{Jm}^{-2}$, slightly under the graphite value of $0.295 \mathrm{Jm}^{-2} /$ layer.

We can also estimate the energy of exfoliaton through a similar procedure. Here we remove a single layer of graphene from the surface of a bulk, while keeping the other layers fixed in position (no relaxation), so that the distances between layers starting at the top are $D, D_{0}, D_{0}, \ldots$ where $D_{0}$ is the bulk graphite interlayer distance. We first perform an LDA calculation, using four layers of which three are fixed in place at spacing $D_{0}$ and the 
TABLE II. Interlayer distance $D_{0}, C_{33}$ elastic coefficient, binding energy $\epsilon$ and peak force $F_{p}$ of graphitic structures. All theory values calculated for this work except $\epsilon$ for graphite from Ref. 19 . Elastic coefficient $C_{33}$ is scaled by two for bigraphene and exfoliation to allow direct comparison with graphite.

\begin{tabular}{|c|c|c|c|c|c|}
\hline Structure & Method & $D_{0}[\AA]$ & $C_{33}[\mathrm{GPa}]$ & $\epsilon\left[\mathrm{Jm}^{-2}\right]$ & $F_{p}[\mathrm{GPa}]$ \\
\hline \multirow[t]{4}{*}{ Graphite } & Expt & $3.34^{a}$ & $36-41^{b}$ & $0.15-0.35^{c \dagger}$ & - \\
\hline & $\mathrm{RPA}$ & 3.334 & 36.1 & $0.295^{\dagger}$ & 1.9 \\
\hline & LDA & 3.32 & 31.3 & $0.16^{\dagger}$ & 1.4 \\
\hline & $\mathrm{LDA}+\mathrm{C}$ & 3.334 & 36.1 & $0.295^{\dagger}$ & 1.7 \\
\hline \multirow[t]{2}{*}{ Bigraphene } & LDA & 3.32 & 29 & $0.15^{\sharp}$ & 1.4 \\
\hline & $\mathrm{LDA}+\mathrm{C}$ & 3.32 & 35 & $0.27^{\sharp}$ & 1.6 \\
\hline \multirow[t]{2}{*}{ Exfoliation } & LDA & 3.32 & 27 & $0.15^{\sharp}$ & 1.3 \\
\hline & $\mathrm{LDA}+\mathrm{C}$ & 3.31 & 36 & $0.31^{\sharp}$ & 1.7 \\
\hline
\end{tabular}

${ }^{a}$ Ref. $28,{ }^{b}$ Refs. $29-32,{ }^{c}$ Refs. 33-36,

$\dagger^{\dagger}\left[\mathrm{Jm}^{-2}\right]=0.0061 \epsilon[\mathrm{meV} /$ Atom $] \sharp \epsilon\left[\mathrm{Jm}^{-2}\right]=0.0122 \epsilon[\mathrm{meV} /$ Atom $]$ (see Sec. II for details)

fourth is varied to $D$. This allows us to determine $U_{\mathrm{ex}}^{\mathrm{LDA}}(D)$. To calculate the dispersion correction, we use the bigraphene correction from all other layers as follows:

$$
\Delta U_{\mathrm{ex}}(D) \approx \sum_{n=0}^{\infty} \Delta U_{\mathrm{bi}}\left(D+n D_{0}\right)
$$

which is added to an LDA calculation with an energy divided by the number of surface atoms ie. four for AB graphite.

While the part of $(17)$ involving $U^{(4)}$ is likely to be correct, the asymptotically dominant $U^{(3)}$ component is invalid for $D \gg D_{0}$, as it predicts $U(D) \propto D^{-2}$ rather than the correct ${ }^{26}$ $U(D) \propto D^{-3} \log \left(D / D^{\prime}\right)$ [where $D^{\prime}$ is a constant of $O(\AA)$. However, in the pre-asymptotic intermediate region $5 \AA \lesssim D \lesssim 10 \AA$ where the $\pi_{z}$ to $\pi_{z}^{*}$ dispersion is a significant fraction of the total potential, but where higher order contributions must also be considered it is a reasonable approximation. Indeed in this region errors are under $0.4 \mathrm{meV} /$ Atom compared to theory.

Results for exfoliation are plotted with their bigraphene counterparts in Figure 3. We use a unit cell of four layers: with inter-layer distances $D_{0}, D_{0}$ and $D$ (where $D_{0}=3.334 \AA$ ). 
These are placed in a supercell with $\vec{a}_{3}=39.38 \AA(0,0,1)$ so that the vaccuum length is always greater than $13 \AA$. Other parameters are the same as for the bigraphene calculations.

The LDA predicts a binding distance $D_{0}^{\mathrm{LDA}}=3.32 \AA$, decreased to $3.31 \AA$ via the dispersion corrections. There are no reliable experimental or high-level theory results to compare with. Along with the shorter inter-layer distance, the effective elastic modulus is increased from $C_{33}^{\mathrm{LDA}}=27 \mathrm{GPa}$ to $C_{33}=36 \mathrm{GPa}$. Finally, the LDA exfoliation energy is predicted to be $0.15 \mathrm{Jm}^{-2}$ or $12 \mathrm{meV} /$ Atom (where we divide only by the number of surface atoms - four for the $\mathrm{AB}$ unit cell), while the dispersion correction increases this to a physically reasonable 0.31 $\mathrm{Jm}^{-2}$ or $25 \mathrm{meV} /$ Surface atom, around $6 \%$ or $0.015 \mathrm{Jm}^{-2}$ greater than the bulk layer binding energy of a graphite layer, and $0.04 \mathrm{Jm}^{-2}$ greater than the bilayer binding energy. This is in good agreement with Björkman et al. ${ }^{14}$ who also found (see Figure 4 of their work) a $0.04 \mathrm{Jm}^{-2}$ difference between the exfoliation and bilayer binding energies of graphite via a different theoretical approach.

Results for graphite, bigraphene and exfoliation are presented together in Table II for easy comparison. In addition to the already defined $D_{0}, C_{33}$ and $\epsilon$ we also include the "peak force" $F_{p}$ in each geometry. Here, we calculate $F(D)=d E(D) / d D$ and its maximum is $F_{p}$ ie. $F_{p}=\sup \{F(D)\}$. Compared to other properties of the energy curve the error on $F(D)$ is quite high at around $10 \%$. This is because the force depends on small differences in the energy at distances $D \approx 4>D_{0}$ where sensitivity to numerical parameters such as the $\vec{k}$-grid is high. The difference of the peak force with and without dispersion correction is expected to be more accurate due to cancellation of errors.

\section{INTERCALATED GRAPHITE}

We now test the theory on lithium intercalated graphite in the $A \alpha$ configuration. $\mathrm{LiC}_{6}$ is a well-studied material (see e.g. the review of Dresselhaus and Dresselhaus, ${ }^{46}$ and Refs. $47-$ 49), and the binding is mostly chemical. We note that the graphene sheets of $\mathrm{LiC}_{6}$ are, in fact, doped to become metallic so that the plasmon-dispersions are changed from graphitic to metallic and the unusual part of the vdW potential is changed. However, at the intermediate distances of interest, the contribution of the metallic/graphitic transitions is a very small part of the total dispersion, and we thus use (3) unchanged. For larger $D$ this approximation would break down. 
FIG. 4. Energy of $\mathrm{LiC}_{6}$ as a function of $D$ in the LDA and LDA with vdW dispersion corrections.

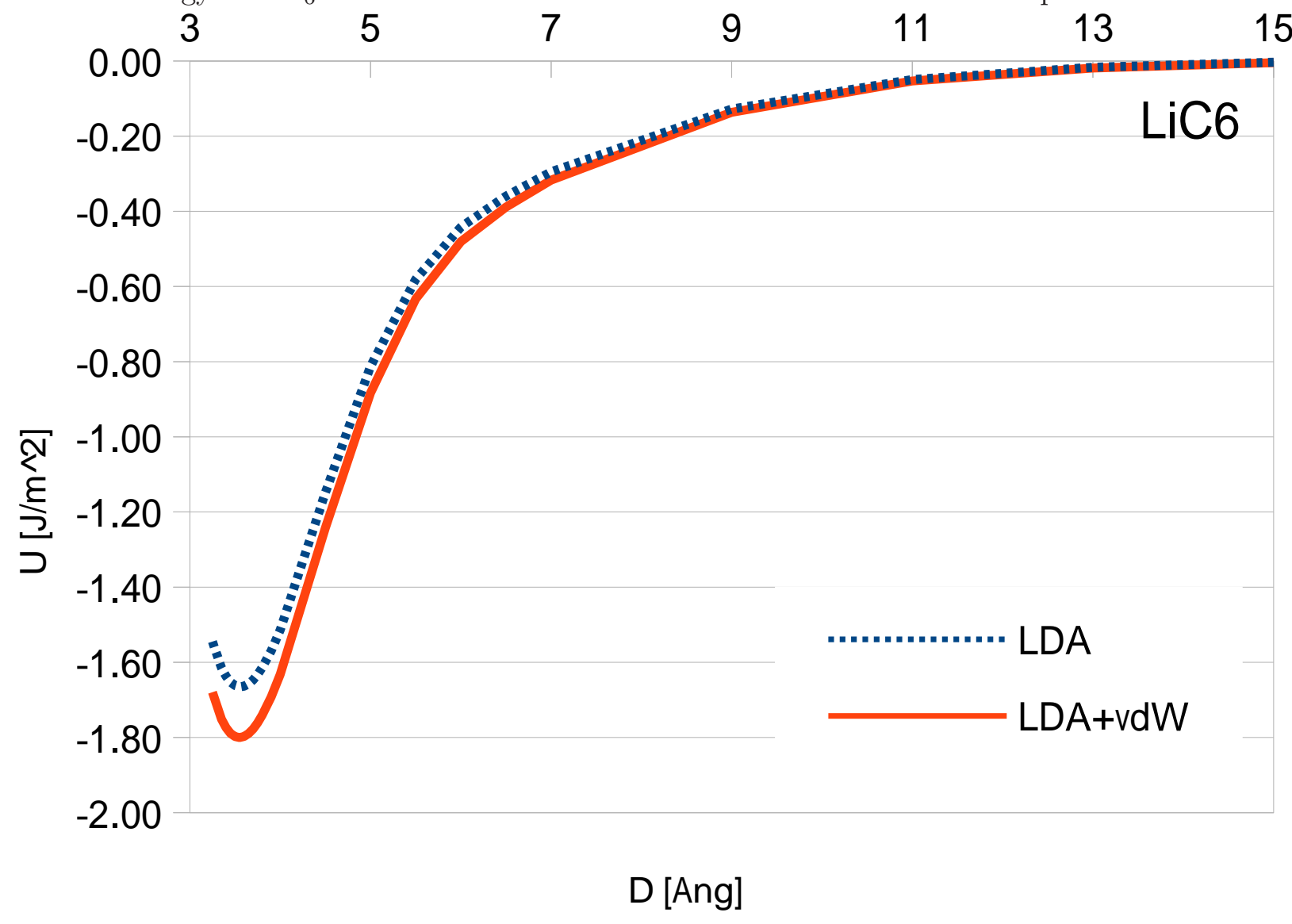

We evaluate $\mathrm{LiC}_{6}$ using default parameters in VASP, and with an $8 \times 8 \times 8 \vec{k}$-grid including the $\Gamma$ point. The inplane $\mathrm{C}-\mathrm{C}$ bond length is set to its experimental value $a_{0}=1.44 \AA$. Results are presented in Figure 4 with and without corrections. Like bigraphene, we find little change to the binding distance of $\mathrm{LiC}_{6}$ at $D_{0}=3.56 \AA$, comparable with the experimental ${ }^{50}$ value $3.70 \AA$. Assuming no relaxation in the planar cell, the 'straight' binding energy per unit area of an intercalate $\mathrm{XC}_{n}$ is $\epsilon_{b}=E_{\mathrm{XC}_{n}}\left(D_{0}\right) / A_{\mathrm{XC}_{n}}-\left(E_{\mathrm{gr}}(\infty)+\frac{2}{n} E_{\mathrm{X}}\right) / A_{\mathrm{gr}}$, where $A_{\mathrm{gr}}$ is the area of the graphene unit cell with two atoms at their intercalated distance. For $\mathrm{LiC}_{6}$ this increases from $\epsilon_{b}^{\mathrm{LDA}}=280 \mathrm{meV} /$ Atom $=1.67 \mathrm{Jm}^{-2}$ to $\epsilon_{b}=301 \mathrm{meV} /$ Atom $=1.80 \mathrm{Jm}^{-2}($ where we divide by the number of carbon atoms - six for the unit cell of $\mathrm{LiC}_{6}$ ). The elastic constant $C_{33}$ of $\mathrm{LiC}_{6}$ is increased slightly from $74 \mathrm{GPa}$ to $76 \mathrm{GPa}$ which compares well with experiment where $^{50-52} C_{33}=79 \pm 10 \mathrm{GPa}$. As one expects the model correction makes little difference to the mechanics or energetics of the intercalate, because dispersion forces are not dominant in the binding. 


\section{CONCLUSIONS}

In this work we presented an interlayer distance $D$-dependent, simple, parametrised model potential of graphite dispersion energies, and total graphite binding energies valid for distances from just inside contact to infinity. The model can be employed on its own or as a correction to standard DFT (LDA/GGA) calculations to improve their energetics. Geometry dependent corrections were then introduced to deal with bigraphene and graphite exfoliation. As a correction the model takes the form ${ }^{21}$

$$
\Delta U(D)=f\left(\frac{D}{D_{0}}-1\right)\left[U^{\mathrm{vdW}}-U^{\mathrm{DFT}}(D)\right]
$$

for graphite, and the geometry adapted forms (16) for bigraphene and (17) for exfoliation. It includes models of the dispersion [eqn (3)] and DFT [eqn (7)] energetics, interpolated by a fitting function [eqn (10)]. Parameters for all functions were determined by fitting the model to high-level RPA $a b$ initio theory results and the theoretical asymptotic dispersion of graphite, and are summarised in Table I.

The model was used to predict the binding energy of bigraphene and exfoliation energy of graphite, with results in good agreement with previous theory. Layers of bigraphene were found to be marginally less bound at $0.27 \mathrm{Jm}^{-2}$ than those of graphite at $0.295 \mathrm{Jm}^{-2}$, while the energy of exfoliation $0.31 \mathrm{Jm}^{-2}$ was found to be slightly larger. Additionally, the model was tested on stage 1 lithium intercalated graphite and found to make only a small change to the energetics, as appropriate.

We believe that the models presented in this paper can provide a simple way of improving ab initio calculations of graphite without resorting to extremely expensive methods such as ACFD-RPA. The dispersion modelling and fitting technique employed here can also be adapted to other layered materials for which conventional modelling techniques struggle due to the presence of coupled plasmons. Our non-pairwise model could also be employed alongside existing vdW approaches such as the DFT-D*9-11 or TS $^{8}$ functionals that account for pairwise atomistic dispersion, provided $\mathrm{C}-\mathrm{C}$ interactions are ignored in the pairwise approach to avoid double counting. 


\section{ACKNOWLEDGMENTS}

TG and JFD were supported by Australian Research Council Discovery Grant DP1096240. JFD was also supported by a visiting position at Université de Lorraine. SL acknowledges financial support from the Université de Lorraine through the program "Soutien à la dimension internationale de la recherche".

\section{Appendix A: Bigraphene model}

The parametrisation given in equations (12)-(14) can be shown to be equally valid for bigraphene as for graphite. However, parameters such as $C_{4}^{\text {bi }}$ and $D_{s}^{\text {bi }}$ require RPA fitting to be determined, and energy results are not available for the case of bigraphene.

Energy data for $U^{(3)}$ and $U^{\text {DFT }}$ are available, and fits show that the variation in $D_{c}$, $\phi_{c}, \tilde{D}$ and $\tau$ are minimal when we change from a graphite to bigraphene geometry. The parameters $C_{3}, C_{4}$ and $M_{0}$ are, unsurprisingly, strongly geometry dependent, as each is proportional to the energetics which vary with the proximity of other layers. We thus assume that only those three parameters are changed from their graphite values, and that the bigraphene parametrisation can be described by the graphene parameters and additional geometry scaling factors $G_{3}, G_{4}$ and $G_{L}$ such that eg. $C_{3}^{\text {bi }}=G_{3} C_{3}$.

To determine $G_{3}$ we use the theoretical values of $C_{3}$ and $C_{3}^{\text {bi }}$. Here $C_{3}$ is calculated through Eq. 12 of Ref. 27. To determine $C_{3}^{\text {bi }}$ we modify Eq. 12 of Ref. 27 by replacing the graphite screened response integral $\left[\int_{0}^{1} \mathrm{~d} \lambda\left\{\mathcal{F}_{\infty}(C)-\mathcal{F}(C)\right\}\right]$ by the bigraphene screened response integral $\left[\log \frac{1-2 C}{1-2 C+C^{2}}\right]$. Evaluating this integral with a cutoff energy of $5 \mathrm{eV}$ also allows us to show that $D_{c}^{\text {bi }}$ and $\phi_{c}^{\text {bi }}$ change only a little from their graphite values. The factor is thus $G_{3}=C_{3} / C_{3}^{\text {bi }} \approx 0.455$.

In the absence of numerical RPA data, to calculate $G_{4}$ we rely on the argument that the 'usual' dispersion terms are additive. That is that the total dispersion energy is given by the sum over the dispersion between all layer pairs, where all interactions are of form $U=C_{4}^{\prime} / D^{4}$. For bigraphene this is $U_{\mathrm{bi}}^{(4)}=C_{4}^{\prime} / D^{4} \equiv C_{4}^{\mathrm{bi}} / D^{4}$ so that we can set $C_{4}^{\prime}=C_{4}^{\mathrm{bi}}$. For graphite, each layer interacts with two other layers at a distance $n D$ where $n \in\{1 \ldots \infty\}$. Thus $U^{(4)}=\sum_{n=1}^{\infty} C_{4}^{\mathrm{bi}} /(n D)^{3}=2 \zeta(4) C_{4}^{\mathrm{bi}} / D^{3}$ and $G_{4}=1 /[2 \zeta(4)] \approx 0.462$.

Finally, while the DFT curve can be fully re-parametrised, for simplicity we treat it in 
the same way. Here we recognise that the local interactions are approximately halved as there are no next-nearest neighbour interactions due to the exponential decay of the LDA or GGA. Thus we set $U_{\mathrm{bi}}^{\mathrm{DFT}}(D) \approx \frac{1}{2} U^{\mathrm{DFT}}(D)$ and $G_{L}=\frac{1}{2}$.

\section{REFERENCES}

1 W. Kohn and L. J. Sham, Phys. Rev. 140, A1133 (1965).

2 J. P. Perdew, "Electronic structure of solids '91," (Akademie Verlag, Berlin, 1991).

3 J. P. Perdew and Y. Wang, Phys. Rev. B 45, 13244 (1992).

4 J. P. Perdew, K. Burke, and M. Ernzerhof, Phys. Rev. Lett. 77, 3865 (1996).

5 H. Rydberg, B. I. Lundqvist, D. C. Langreth, and M. Dion, Phys. Rev. B 62, 6997 (2000).

6 H. Rydberg, M. Dion, N. Jacobson, E. Schröder, P. Hyldgaard, S. I. Simak, D. C. Langreth, and B. I. Lundqvist, Phys. Rev. Lett. 91, 126402 (2003).

7 M. Dion, H. Rydberg, E. Schröder, D. C. Langreth, and B. I. Lundqvist, Phys. Rev. Lett. 92, $246401(2004)$.

8 A. Tkatchenko and M. Scheffler, Phys. Rev. Lett. 102, 073005 (2009).

9 S. Grimme, Journal of Computational Chemistry 25, 1463 (2004).

10 S. Grimme, J. Comp. Chem. 27, 1787 (2006).

11 S. Grimme, J. Antony, S. Ehrlich, and H. Krieg, The Journal of Chemical Physics 132, 154104 (2010).

12 O. A. Vydrov and T. Van Voorhis, Phys. Rev. A 81, 062708 (2010).

13 T. Björkman, A. Gulans, A. V. Krasheninnikov, and R. M. Nieminen, Journal of Physics: Condensed Matter 24, 424218 (2012).

14 T. Björkman, A. Gulans, A. V. Krasheninnikov, and R. M. Nieminen, Phys. Rev. Lett. 108, $235502(2012)$.

15 J. F. Dobson and T. Gould, Journal of Physics: Condensed Matter 24, 073201 (2012).

16 J. F. Dobson, A. White, and A. Rubio, Phys. Rev. Lett. 96, 073201 (2006).

17 H. Eshuis, J. E. Bates, and F. Furche, Theoretical Chemistry Accounts 131, 1 (2012).

18 X. Ren, P. Rinke, C. Joas, and M. Scheffler, Journal of Materials Science 47, 7447 (2012). 
19 S. Lebègue, J. Harl, T. Gould, J. G. Ángyán, G. Kresse, and J. F. Dobson, Phys. Rev. Lett. 105, $196401(2010)$.

20 J. Harl and G. Kresse, Phys. Rev. Lett. 103, 056401 (2009).

21 M. Hasegawa and K. Nishidate, Phys. Rev. B 70, 205431 (2004).

22 M. Hasegawa, K. Nishidate, and H. Iyetomi, Phys. Rev. B 76, 115424 (2007).

23 L. A. Girifalco and M. Hodak, Phys. Rev. B 65, 125404 (2002).

24 F. Ortmann, F. Bechstedt, and W. G. Schmidt, Phys. Rev. B 73, 205101 (2006).

25 T. Gould, K. Simpkins, and J. F. Dobson, Phys. Rev. B 77, 165134 (2008).

26 T. Gould, E. Gray, and J. F. Dobson, Phys. Rev. B 79, 113402 (2009).

27 T. Gould, J. F. Dobson, and S. Lebègue, Phys. Rev. B 87, 165422 (2013).

28 Y. Baskin and L. Meyer, Phys. Rev. 100, 544 (1955).

29 O. L. Blakslee, D. G. Proctor, E. J. Seldin, G. B. Spence, and T. Weng, Journal of Applied Physics 41, 3373 (1970).

30 W. B. Gauster and I. J. Fritz, Journal of Applied Physics 45, 3309 (1974).

31 N. Wada, R. Clarke, and S. Solin, Solid State Communications 35, 675 (1980).

32 A. Bosak, M. Krisch, M. Mohr, J. Maultzsch, and C. Thomsen, Phys. Rev. B 75, 153408 (2007).

33 L. A. Girifalco and R. A. Lad, The Journal of Chemical Physics 25, 693 (1956).

34 L. X. Benedict, N. G. Chopra, M. L. Cohen, A. Zettl, S. G. Louie, and V. H. Crespi, Chem. Phys. Letters 286, 490 (1998).

35 R. Zacharia, H. Ulbricht, and T. Hertel, Phys. Rev. B 69, 155406 (2004).

36 Z. Liu, J. Z. Liu, Y. Cheng, Z. Li, L. Wang, and Q. Zheng, Phys. Rev. B 85, 205418 (2012).

37 Notably the model used in Ref. 36 produces internal inconsistencies in the elastic coefficient $C_{33}$.

38 S. D. Chakarova-Käck, E. Schröder, B. I. Lundqvist, and D. C. Langreth, Phys. Rev. Lett. 96, 146107 (2006).

39 E. Ziambaras, J. Kleis, E. Schröder, and P. Hyldgaard, Phys. Rev. B 76, 155425 (2007).

40 L. Spanu, S. Sorella, and G. Galli, Phys. Rev. Lett. 103, 196401 (2009).

41 A. Ruzsinszky, J. P. Perdew, J. Tao, G. I. Csonka, and J. M. Pitarke, Phys. Rev. Lett. 109, 233203 (2012).

42 The cutoff off $5 \mathrm{eV}$ is approximately the gap from the Fermi energy to the bottom of the Dirac 
cone. A larger value would cause the theoretical model to include transitions that are not available in real graphite. With the other dispersion terms included, the model is not very sensitive to variations in the energy cutof, at least in the regions of interest.

43 G. Kresse and J. Furthmüller, Phys. Rev. B 54, 11169 (1996).

44 G. Kresse and D. Joubert, Phys. Rev. B 59, 1758 (1999).

45 T. Bučko, J. Hafner, S. Lebègue, and J. G. Ángyán, The Journal of Physical Chemistry A 114, $11814(2010)$.

46 M. S. Dresselhaus and G. Dresselhaus, Advances in Physics 51, 1 (2002).

47 N. A. W. Holzwarth, S. Rabii, and L. A. Girifalco, Phys. Rev. B 18, 5190 (1978).

48 N. A. W. Holzwarth, S. Rabii, and L. A. Girifalco, Phys. Rev. B 18, 5190 (1978).

49 K. R. Kganyago and P. E. Ngoepe, Molecular Simulation 22, 39 (1999).

50 H. Zabel, A. Magerl, and J. J. Rush, Phys. Rev. B 27, 3930 (1983).

51 P. Zhou and J. E. Fischer, Phys. Rev. B 53, 12643 (1996).

52 C. Bindra, V. A. Nalimova, D. E. Sklovsky, W. A. Kamitakahara, and J. E. Fischer, Phys. Rev. B 57, 5182 (1998). 